\title{
DEVELOPMENT OF A BEAM IMAGING SYSTEM FOR THE EUROPEAN SPALLATION SOURCE TUNING DUMP
}

\author{
M. G. Ibison ${ }^{\dagger}$, C. P. Welsch, Cockcroft Institute and University of Liverpool, UK \\ E. Adli, H. Gjersdal, G. Christoforo, University of Oslo, Norway** \\ T. Shea, C. Thomas, ESS, Lund, Sweden \\ D. Naeem, STFC, Daresbury, UK
}

\begin{abstract}
To provide essential beam diagnostics for the spallation target and for the tuning dump of the European Spallation Source (ESS) with its high-power, low-emittance proton beam, optical imaging systems have been developed allowing remote viewing of the beam profiles, using scintillation light from coatings on the proton beam window (PBW) at the accelerator exit and the target entry window (TW), and insertable interceptive screens at the tuning dump (TD).

In this paper, we present the techniques used and the main parameters in the design of the TD system, including the optical configurations modelled in the software Zemax OpticStudio (ZOS). We also present the design principles for reflective optics which can transmit high-quality images, showing the performance of the imaging systems as predicted by ZOS and by prototyping. We take account of the requirements for initial alignment and ongoing maintenance of the optical system.

Studies of the radiation and thermal environments, which impact on sensitive optical components such as cameras and mirrors, are also described, as are mechanical considerations for the vacuum vessels and screen actuators. Finally, comments are made on the applicability of similar optics to diagnostic systems at other neutron sources and accelerators.
\end{abstract}

\section{TUNING DUMP OPTICAL DIAGNOS- TICS: SYSTEM REQUIREMENTS}

The fundamental requirements for the TD diagnostics are summarised in Error! Reference source not found. Beam conditions and the physical environment are specific to the dump line.

\section{Tuning Dump Optical System Parameters}

The Tuning Dump (TD) is designed to receive the ESS beam during initial commissioning and LINAC tune-up, whenever the beam is to be studied without sending it to the target. The dump can safely handle short proton pulses, and also full pulses but at reduced rate.

It was originally envisaged that a single imaging system located at the termination of the dump beam-line should be capable of viewing the beam incident on the dump face, in a similar way to beam-on-target imaging, as well as its profile immediately before its exit from the beam-pipe.

Table 1 TD system requirements - main parameters

\begin{tabular}{|l|l|l|}
\hline \multicolumn{1}{|c|}{ Parameter } & \multicolumn{1}{c|}{ Value } & \multicolumn{1}{c|}{ Origin } \\
\hline Field of View & $\begin{array}{l}\text { Max. } \\
\text { possible }\end{array}$ & $\begin{array}{l}\text { Full beam-pipe diameter } \\
=250 \mathrm{~mm}\end{array}$ \\
\hline $\begin{array}{l}\text { Limiting Ap- } \\
\text { ertures }\end{array}$ & $\begin{array}{l}200 \mathrm{~mm} \\
100 \mathrm{~mm}\end{array}$ & $\begin{array}{l}\text { Vessel viewports } \\
\text { Camera penetration* }\end{array}$ \\
\hline $\begin{array}{l}\text { Beam Size } \\
\text { (nominal) }\end{array}$ & $\begin{array}{l}1.6 \mathrm{~cm} \\
(\mathrm{rms})\end{array}$ & $\begin{array}{l}\text { Beam dynamics simula- } \\
\text { tion [1] }\end{array}$ \\
\hline Resolution & $\leq 1 \mathrm{~mm}$ & Beam profile \\
\hline $\begin{array}{l}\text { Max Average } \\
\text { Power }\end{array}$ & $12.5 \mathrm{~kW}$ & $\begin{array}{l}\text { Use Case: 'Slow Tuning } \\
\text { Beam' [1] }\end{array}$ \\
\hline \multicolumn{2}{|c|}{ *see Figure 14 }
\end{tabular}

\section{Modified Tuning Dump System Requirements}

It is not now planned that the dump face will receive the luminescent coating which could have generated an image. The requirement for the TD system has therefore been simplified to providing imaging of the beam transverse profile at two or more locations upstream of the dump itself. These will be separated longitudinally to retain the capability for beam divergence assessment. The system is also required to be able to image a beam occupying any part of the full physical beam-pipe aperture at its exit.

\section{OPTICS DESIGN APPROACH}

Because of the hostile radiation environment in the TD tunnel area, a system based on all-reflective optics has been selected, rather than lenses or optical fibres which are known to suffer rapid damage [2]. Nevertheless, some degradation of components close to the proton beam is to be expected over time.

The industry-standard optical system design software tool Zemax OpticStudio [3] has been used to perform all design work, with theoretical values as initial parameters. While not essential, use of Zemax has assisted with integration of the optics with beam-line CAD models. Pure ray-optics modelling was considered to be sufficiently accurate, as diffractive effects may be neglected. 


\section{Tuning Dump Optics Design}

Initial concepts explored for TD imaging included a 3curved-mirror system based on aberration compensation principles [4], resembling the optics previously designed for PBW/TW imaging [5][6][7], and also an optical fibre solution with cameras located remotely. The multi-mirror option was considered unnecessarily complex for the minor gain in field-of-view, while fibres would be too susceptible to radiation damage and have inferior optical characteristics, in addition to cost and availability issues.

The final design selected has a simple 'periscope' configuration comprising 2 plane mirrors, which combines acceptable image quality with flexible camera positioning.

To meet the original requirement, which envisaged the imaging of the beam as it entered the dump face, an early design was devised with a composite screen/mirror system to image the beam on the screen front surface as it passed through, while simultaneously collecting a reflected image of the beam on dump by using the rear surface of the screen as a mirror. With the relaxation of the dump imaging requirement, it has become possible to simplify the system to a plain screen, eliminating the need for rear mirroring. The optical components comprising the model therefore include:

- the object (screen intercepting proton beam)

- the viewport in the vacuum vessel

- $\quad 1^{\text {st }} 45^{\circ}$ mirror (outside the viewport)

- $\quad 2^{\text {nd }} 45^{\circ}$ mirror (on ray-path from $1^{\text {st }}$ mirror)

- $\quad$ imaging lens and camera

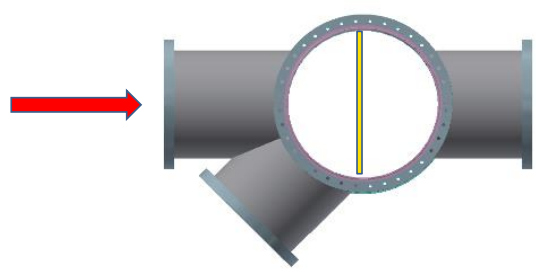

Figure 1 Screen in centre of imaging vessel - plan view. Proton beam direction is indicated by the arrow; the screen orientation shown is at $90^{\circ}$ to the beam.

\section{Photon Sources for Imaging}

The primary light source for TD imaging will be a 'Chromox' ceramic screen, although an alternative with a thermally-sprayed luminescent coating, most probably $\mathrm{Cr}$ doped alumina on an aluminium backing, will also be fitted. Screen materials are excited into photon emission by

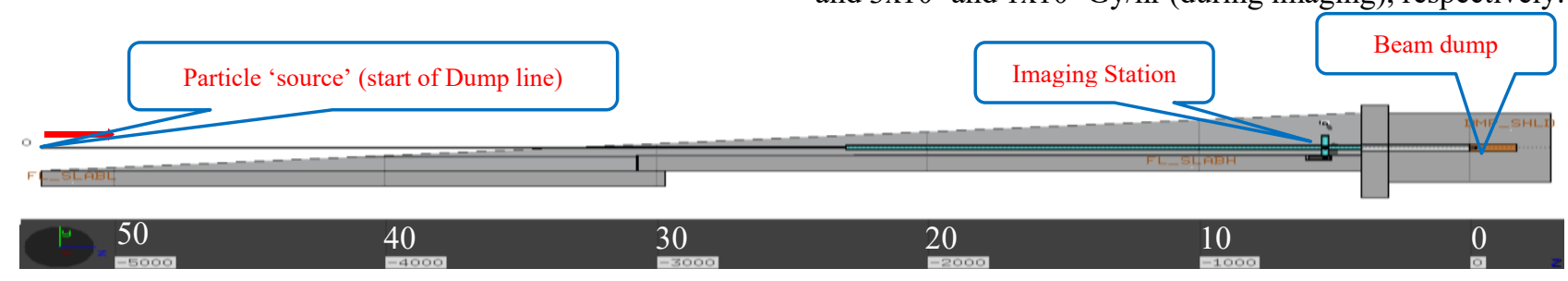

Figure 2 FLUKA model of the dump line tunnel (elevation shown from RHS); beam from L to R. Dimensions in m. the energy of incident protons. Studies are ongoing to identify materials which could improve on the standard widelyused and commercially-available 'Chromox' [8]; however, these must give adequate photon yield per proton, emission spectrum, lifetime \& linearity, while maintaining their properties after the heat exposure of the spraying process.

\section{STUDIES OF PHYSICAL ENVIRONMENT}

The physical environment in which the optical systems must function has an effect not only on their performance, in terms of image quality and stability, but also on the useful lifetime of their components. This applies to the effects of both heating by deposition of energy from the beam and also damage at the atomic level from particle collisions.

\section{Tuning Dump - Radiation Environment}

The dump line with its vacuum vessels, the dump structure itself, and the enclosing shielded tunnel, have been modelled using the Monte-Carlo radiation transport code FLUKA [9]. By applying the expected beam parameters at the dump and the estimated utilisation, based on the ESS operating schedule, to the outputs of the model, the total radiation dose per year has been calculated, at locations of interest in the vicinity of the imaging vessel. The objective was to find positions for the cameras which while providing the required field of view would ensure an adequate useful lifetime, before radiation damage to the CCD sensor compromised the image quality unacceptably.

\section{Camera Radiosensitivity}

Studies have been made both of dose and of neutron or proton fluence, as related to observable effects expected in image quality or system performance, including:

- permanent damage to sensor pixels

- 'upsets' to camera electronics, recoverable after reset

- $\quad$ irrecoverable damage causing imaging failure

Based on these sources [10], and the expected major annual shutdown schedule during which degraded optical elements may be replaced, an arbitrary target dose of $<20$ Grays/year has been set for selection of the imaging camera locations, with any associated shielding. At a fraction of this dose, no detectable effects are expected.

A number of alternative, specifically 'radiation-hard' camera solutions were considered, such as the older 'Vidicon' tube type which are still available (e.g. Mirion), or CID sensor types (e.g. Thermo Scientific). These have quoted operational dose limits of $2 \times 10^{6}$ and $3 \times 10^{4}$ Gy (lifetime), and $3 \times 10^{4}$ and $1 \times 10^{3} \mathrm{~Gy} / \mathrm{hr}$ (during imaging), respectively. 
However, for optimum image quality and choice of supply, an adequately shielded standard scientific CCD camera is preferred; details of the selected type are listed in Table 5. As occasional single event upsets from radiation are to be expected, the camera control system must allow rapid reset by remote power-cycling.

Although no other critical component is as radiosensitive as the cameras, dose assessments have also informed the selection - or avoidance - of such susceptible materials as elastomer vacuum seals and actuator flexible drive belts.

\section{Radiation Transport Modelling with FLUKA}

The FLUKA code is a mature simulation environment for the Monte-Carlo modelling of radiation transport (charged particles, neutrons \& photons) through matter. A model of the ESS tuning dump, the TD beam-line and its shielded tunnel has been created, including all significant geometry and material compositions, as shown in Figure 2. A simulation is run by propagating particles from the start of the beam-line towards the dump and collecting data on the history of each primary particle as it interacts, possibly producing secondary particles which are also tracked. As events in the regions of particular interest were rare, a very large number of primaries had to be followed; typically this was $5 \times 10^{5}$ to $10^{6}$ for the TD geometry, for statistically meaningful results to be obtained. Following FLUKA guidelines, five repeated independent runs were performed for each model, allowing an estimation of the associated variance.

The external viewer program 'SimpleGeo' has proved useful for preparing detailed 3-D geometry images and for the plotting of particle tracks, based on FLUKA data files; it also provides a CAD modelling interface.

\section{Camera Radiation Dose}

For the TD system, the absorbed dose was recorded in those regions proposed for locating the imaging cameras. Dose is estimated by FLUKA per primary particle, the total number of which must be calculated from the projected beam current and the annual time for beam on dump, based on the equation:

$$
\begin{aligned}
\text { Protons per year } & =0.5 \times t_{S} \times 3600 \times \frac{I_{P}}{e} \\
= & 3.54 \times 10^{19}
\end{aligned}
$$

where annual machine study time $\mathrm{t}_{\mathrm{S}}=500 \mathrm{~h}$; time-ondump fraction $($ estimated $)=0.5$; beam current $($ mean $) \mathrm{I}_{\mathrm{P}}=$ $6.3 \mu \mathrm{A} ; \mathrm{e}=$ electronic charge

It was also demonstrated that a large fraction of the dose received by an unshielded camera is due to particles scattered by the imaging screen itself; with the screen removed, only that radiation escaping from the dump entrance makes a contribution. Figure 3 shows the imaging vessel in the dump-line tunnel, with the proton beam propagating from $\mathrm{L}$ to $\mathrm{R}$. Tracks of different types of scattered particle are indicated by colour. On the top picture, the screen is inserted into the beam; on the bottom it is removed. Each run is for equal numbers of primary protons.

As new candidate locations were explored, the FLUKA model was developed progressively by adding the required geometry, so that doses at multiple camera positions could be compared during the same run.

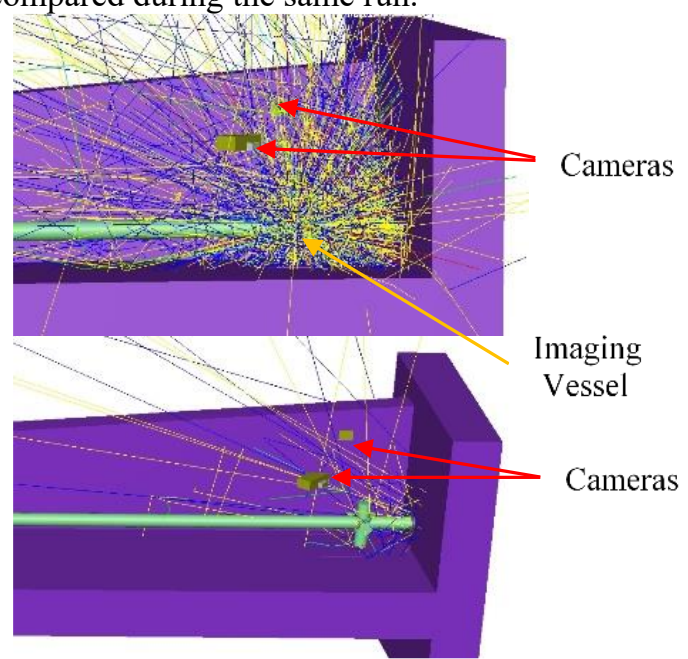

Figure 3 FLUKA particle tracking displayed in 'SimpleGeo', with screen inserted (top) and removed (bottom). Camera locations shown are unshielded. Beam is L. to R.

Table 2 Camera doses for different locations. Camera 1 to $\mathrm{L}$ side, Camera 2 to R. Doses in Gy/year; errors at $\pm 1 \sigma$.

\begin{tabular}{|l|l|l|}
\hline \multicolumn{1}{|c|}{ Location } & Camera 1 & Camera 2 \\
\hline $\begin{array}{l}\text { Shielded Bunker on Tunnel } \\
\text { Floor, 5cm concrete lid }\end{array}$ & $700 \pm 150$ & $380 \pm 90$ \\
\hline $\begin{array}{l}\text { Shielded Bunker, thicker } \\
\text { cover plate, 7.5cm concrete }\end{array}$ & $430 \pm 60$ & $650 \pm 140$ \\
\hline $\begin{array}{l}\text { Shielded Trench excavated } \\
\text { in Floor Slab, 7.5cm cover }\end{array}$ & $325 \pm 350$ & $275 \pm 350$ \\
\hline $\begin{array}{l}\text { High-Level } \uparrow, \text { otherwise } \\
\text { layout as for Bunker case }\end{array}$ & $100 \pm 150$ & $40 \pm 70$ \\
\hline $\begin{array}{l}\text { High-Level†, further dis- } \\
\text { placed from beam axis }\end{array}$ & $180 \pm 290$ & $24 \pm 22$ \\
\hline $\begin{array}{l}\text { High-Level } \uparrow, \text { down-looking } \\
\text { onto 1 }\end{array}$ mirrors & $120 \pm 60$ & $55 \pm 30$ \\
\hline $\begin{array}{l}\text { High-Level, horizontal in } \\
\text { wall 'niches' }\end{array}$ & $\begin{array}{l}45 \pm 20 \\
(5 \pm 6) *\end{array}$ & $15 \pm 9$ \\
\hline $\begin{array}{l}\text { High-Level, set well back } \\
\text { into walls }\end{array}$ & $15 \pm 25$ & $12 \pm 7$ \\
\hline High-Level, in 1.5m hole & N/A & undetectable \\
\hline
\end{tabular}

*Note: Doses with screen removed from beam; see Figure 3. + High-level cameras unshielded, unless in walls. In Table 2, results show that with cameras located close to or just under the beam-line, addition of shielding alone cannot sufficiently reduce the dose to the target. The combination of height and the optimal use of the existing shield wall thickness has provided a solution. Drilling restrictions limit the size of hole, primarily due to the wall 


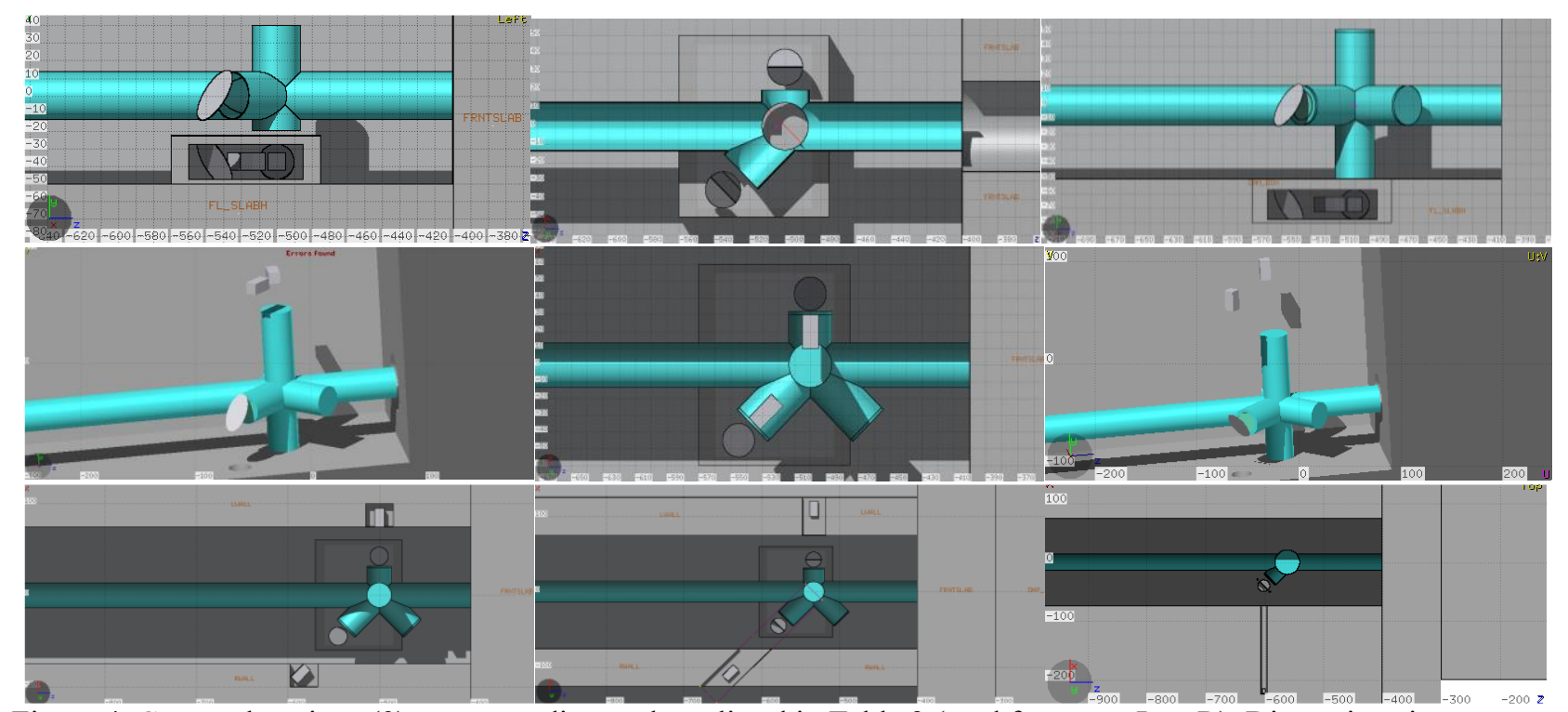

Figure 4 Camera locations (9) corresponding to those listed in Table 2 (read from top, L to R). Dimensions in $\mathrm{cm}$.

reinforcement, but the hole will have a minimum diameter of $100 \mathrm{~mm}$; its depth will not exceed $1.5 \mathrm{~m}$. It should also be noted that due to changed imaging requirements (see Modified Requirements), only a single camera will in fact be deployed; in Table 2, 'Camera 1' and 'Camera 2' refer to locations on opposite side of the beam-line. Figure 4 illustrates the 9 locations listed: cameras are represented as grey cuboids in the vicinity of the vessel.

\section{Dose from Beam Losses}

When the ESS is operating in 'User' mode, the beam from the LINAC is directed into the target line by dipole magnets in the first bend of the 'dog-leg' section. Some of the protons are lost from the beam in this region and will enter the dump tunnel, as shown in Figure 5, contributing to the total dose received by the imaging cameras. To model these losses, advantage was taken of earlier ESS modelling work to provide the input data files containing full parameter sets (position, direction vectors and energy) for a large set of particles. A customised code module 'SOURCE' was written to read the pre-processed particle data into the existing TD FLUKA model. From this, the dose per source proton at the camera location was obtained, and hence the annual dose derived using the following result:

$$
\begin{aligned}
& \text { Proton Losses to Dump Tunnel per year } \\
& \qquad \begin{array}{l}
=(5300 \times 3600) \times\left(2.52 \times 10^{-3}\right) \\
\times(0.002 \times 0.01) / e \\
=6.00 \times 10^{18} \text { protons }
\end{array}
\end{aligned}
$$

where average beam current (5MW full power beam on target $)=2.52 \mathrm{~mA}$, operating hours $=5300$ per year, and fractional loss rate at the dipole $=0.002 \%$ (assumed).

Doses to the camera in its final selected location as shown in Figure 14 were undetectable in FLUKA simulations.

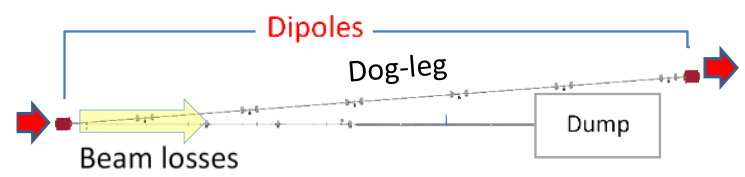

Figure 6 Beam losses from first dipole in the dog-leg section, which enter the dump tunnel. Beam from LINAC enters from $\mathrm{L}$, beam to target leaves to $\mathrm{R}$.

\section{Decay Dose-Rates}

A separate study was made of the dose-rates which would be expected in the vicinity of the imaging station, close to the TD, in the period immediately after beam shut-off, due to the decay of activation products in and around the dump.

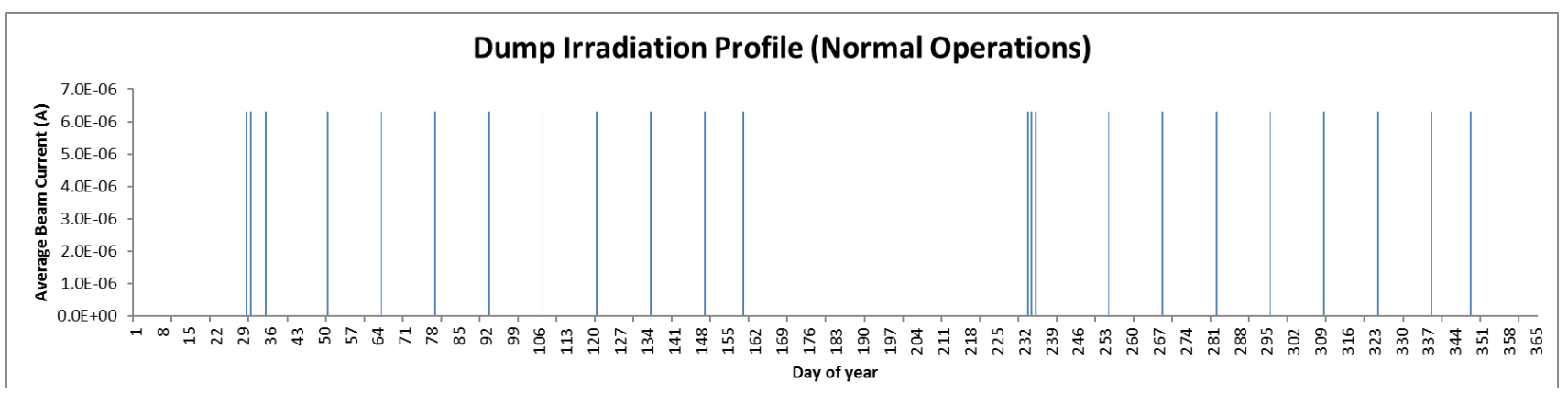

Figure 5 Projected beam-on-dump irradiation profile for 1 full year, after the start of normal user operations. Particles delivered in each period are based on an average beam current $I_{p}=6.3 \mu \mathrm{A}$. 
The pattern of beam-on-dump periods taken from the proposed ESS Operations Schedule, after the initial start-up date, is shown in Figure 6.

FLUKA accepts arbitrary particle irradiation times and rates as input, and provides dose-rate output at any selected decay times after beaming. Dose-rate 2-D profiles in the horizontal plane of the beam are plotted in Figure 7 , for times of 1, 8, 24 and 72 hours after shut-down, at the end of one year's operation.
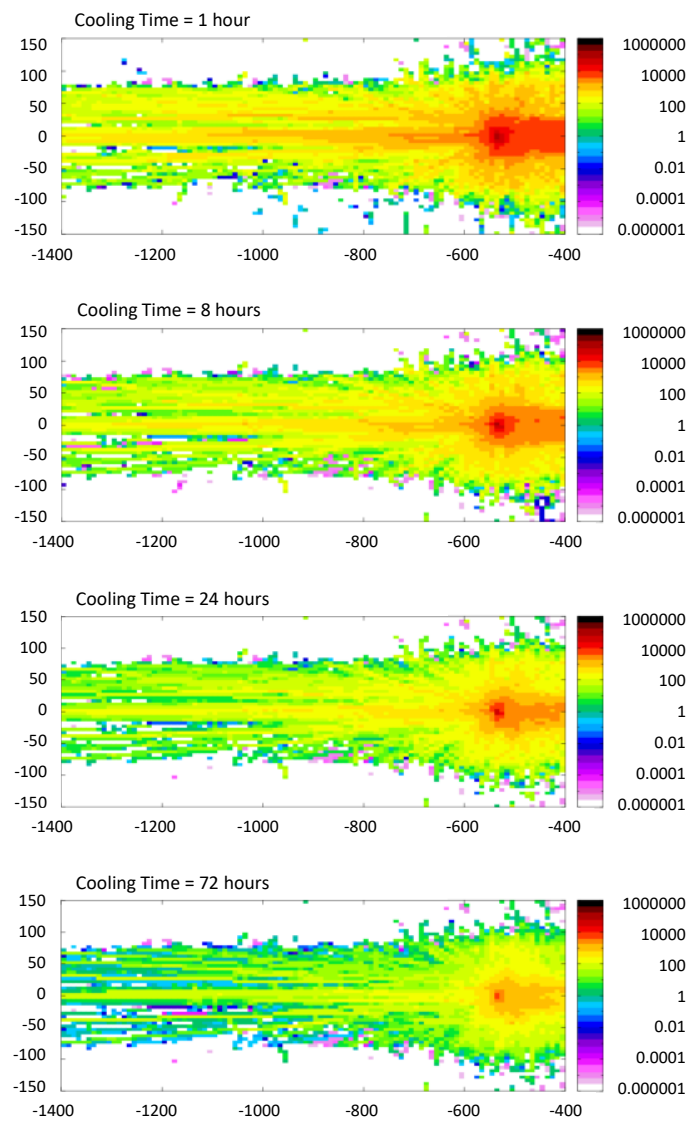

Figure 7 Decay dose-rate plots in horizontal beam plane in the vicinity of the imaging vessel, after $1,8,24, \& 72$ hours' cooling time. Distances in $\mathrm{cm}$, dose-rate in $\mu \mathrm{Sv} / \mathrm{h}$.

The intense spot in the centre-right of Figure 7 is due to the imaging screen; decay in radiation streaming from the activated dump itself (to $\mathrm{R}$ of the plots) is also evident.

An independent study was made by analytical calculation of the activity induced by a $4.5 \mathrm{~cm}$ radius beam of $2 \mathrm{GeV}$ protons via $\mathrm{Cu}(p, \mathrm{x} n)$ reactions [11] in the copper dump cylinder and the subsequent decay of the 21 most important nuclides produced, using the equation [12]:

$$
S_{A}\left(B q g^{-1}\right)=\frac{N_{A} \sigma}{A} \frac{d \varphi}{d t}\left(1-e^{\frac{-t_{i} \ln 2}{t_{\frac{1}{2}}}}\right) e^{\frac{-t_{c} \ln 2}{t_{1}}}
$$

where $\mathrm{N}_{\mathrm{A}}$ is Avogadro's Number, $\sigma$ is the cross-section for the reaction, $\mathrm{A}$ is the nucleon number for $\mathrm{Cu}$ (taken as the atomic weight for natural copper), $\mathrm{d} \phi / \mathrm{dt}$ is the particle fluence rate, $t_{i}$ is the irradiation time, $t_{c}$ is the cooling time, and $t_{1 / 2}$ is the radioactive half-life. This equation gives the activity per gram $S_{A}$ at time $t_{c}$ after the end of the irradiation. The result is converted into the total activity in the volume of the dump, using the density of $\mathrm{Cu}$.

The gamma dose-rate at $4 \mathrm{~m}$ from this source on the beam axis (the approximate location of the imaging vessel), was then estimated. The dose-rate D' is calculated for each significant gamma-ray from [13]:

$$
D^{\prime}=\frac{A n E}{4 \pi}\left(\frac{\mu_{e n}}{\rho}\right)(G)
$$

where $A$ is the nuclide activity, $n$ is the gamma-ray abundance, $E$ is the gamma energy (in J), $\mu_{\mathrm{en}} / \rho$ is the mass energy-absorption coefficient (for air), and $G$ is the 'geometry factor' for a cylindrical source, derived from the expression [14]:

$$
\begin{aligned}
\frac{1}{R_{0}^{2} h}\left\{\left(\frac{h}{2}+z\right) \ln [\right. & \left.1+\frac{R_{0}^{2}}{\left(\frac{h}{2}+z\right)^{2}}\right] \\
& -\left(z-\frac{h}{2}\right) \ln \left[1+\frac{R_{0}^{2}}{\left(z-\frac{h}{2}\right)^{2}}\right] \\
& \left.+2 R_{0}\left[\tan ^{-1} \frac{\frac{h}{2}+z}{R_{0}}-\tan ^{-1} \frac{z-\frac{h}{2}}{R_{0}}\right]\right\}
\end{aligned}
$$

where $\mathrm{R}_{0}$ and $\mathrm{h}$ are the radius and height of the cylinder, and $\mathrm{z}$ is the distance from its centre, on axis.

The contributions are then summed for the total dose-rate.

Table 3 Summary of decay dose-rates from two estimation methods. All dose-rates are quoted in $\mathrm{mSv} / \mathrm{hr}$.

\begin{tabular}{|l|l|l|}
\hline Cooling Time (hours) & 1 & 72 \\
\hline Total Dose-Rate (analytical) & 51.3 & 20.6 \\
\hline Dose-Rate (from FLUKA) & $10-100$ & $1-10$ \\
\hline
\end{tabular}

The results shown in Table 3 are consistent, given the calculation uncertainties, especially as the analytical approach ignores self-shielding by the dump. At the shorter cooling time, FLUKA data will be enhanced by rapidly- decaying local radiation from the screen.

\section{Screen Heating Studies}

As the beam passes through the imaging screen, it deposits some energy as heat, thereby raising the temperature [15]. In the beam-pipe vacuum, only radiative and conductive processes are available to dissipate the heat. Studies were

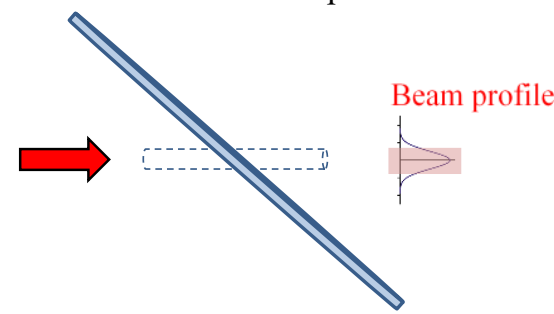

Figure 8 Model of passage of beam through screen, showing 'core' region for thermal analysis ( $1 \sigma$ width). 
carried out into the instantaneous heating effect of a single full ESS proton pulse, assuming no immediate heat removal. In Figure 9, the peak temperature reached in the various layers of a composite screen is plotted against beam size (at $1 \sigma$ ), assuming a Gaussian distribution. The plots labelled 'SCR_xBC' relate to the beam $1 \sigma$ 'core' region of the screen layers, treated as if in thermal isolation from the outer regions.

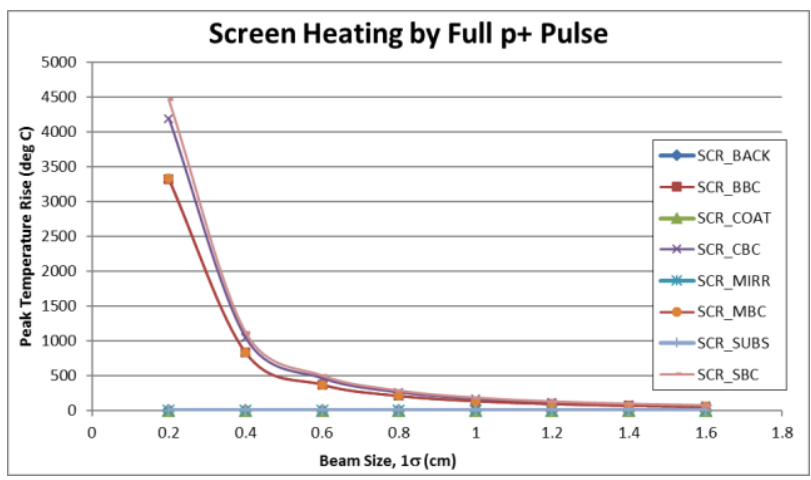

Figure 9 Peak temperature in screen layers vs. beam size, for a single full pulse of $1.114 \times 10^{15}$ protons, at $E_{p}=570$ $\mathrm{MeV}$.

Considering the melting points (MP) of candidate screen materials, these results indicate that a coated aluminium screen $\left(\mathrm{MP}=660^{\circ} \mathrm{C}\right)$ is safe down to a beam size $\sigma_{\mathrm{x}} \geq 0.75$ $\mathrm{cm}$, while a Chromox screen $\left(\mathrm{MP} \sim 2000^{\circ} \mathrm{C}\right)$ could be used at $\sigma_{\mathrm{x}} \geq 0.4 \mathrm{~cm}$.

In comparison, a nominal beam of $\sigma_{\mathrm{x}}=1.6 \mathrm{~cm}$ is predicted to heat the screen materials by a maximum of $70^{\circ} \mathrm{C}$.

\section{SYSTEM PROTOTYPING}

\section{Tuning Dump System}

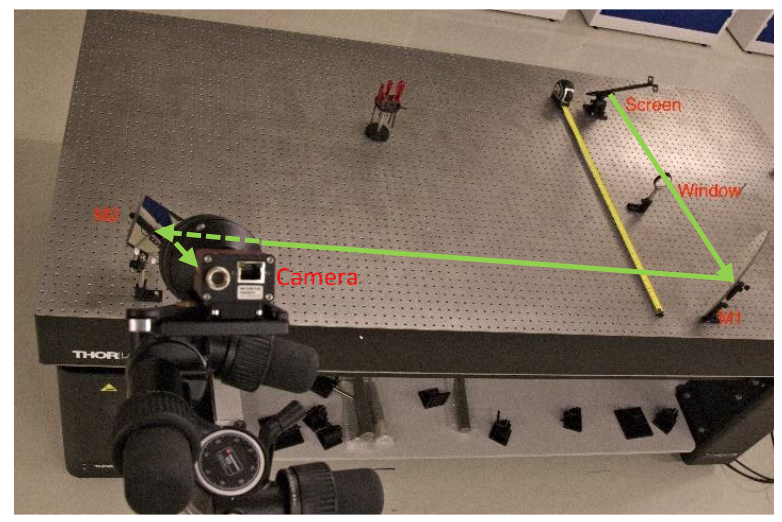

Figure 10 Prototyping with optical table for resolution tests, showing light path to camera via mirrors M1, M2 (looking down on path, camera seen in L. foreground).

At the University of Oslo ( $\mathrm{UiO}$ ) a prototyping facility has been constructed with two large aligned optical tables; these may be used in tandem to model the long path lengths of the ESS optical systems. Prototyping assists in confirming the results of ZEMAX optical simulations, which cannot always reliably incorporate the effects on image quality of realistic errors in optical components. The effects of apertures and limits on fields of view can also be evaluated, and the proposed initial assembly, alignment and maintenance procedures assessed.

\section{PROJECT PLANNING}

The development of the project has been marked by a number of milestones and major reviews, as imaging systems for both the PBW/TW and the TD were developed in parallel:

- Preliminary Design Review (PDR)

- Critical Design Review (CDR)

- System Prototyping

For the TD system, additional review stages have been:

- PDR specifically for the imaging vessel, this being integral to the full TD beam-line

- $\quad$ final CDR and sign-off for the vessel, with a full documented specification

Stages yet to be completed include:

- $\quad$ Placing of orders for components, \& fabrication as per specification

- Delivery \& integration; acceptance testing

- Final installation \& commissioning on site, in 'cold check-out' and ultimately, with beam.

Initial mechanical testing will be integrated with the standard vacuum vessel acceptance process at the STFC Daresbury Laboratory.

\section{DESCRIPTION OF FINAL DESIGN}

\section{Optical Performance of the TD Design}

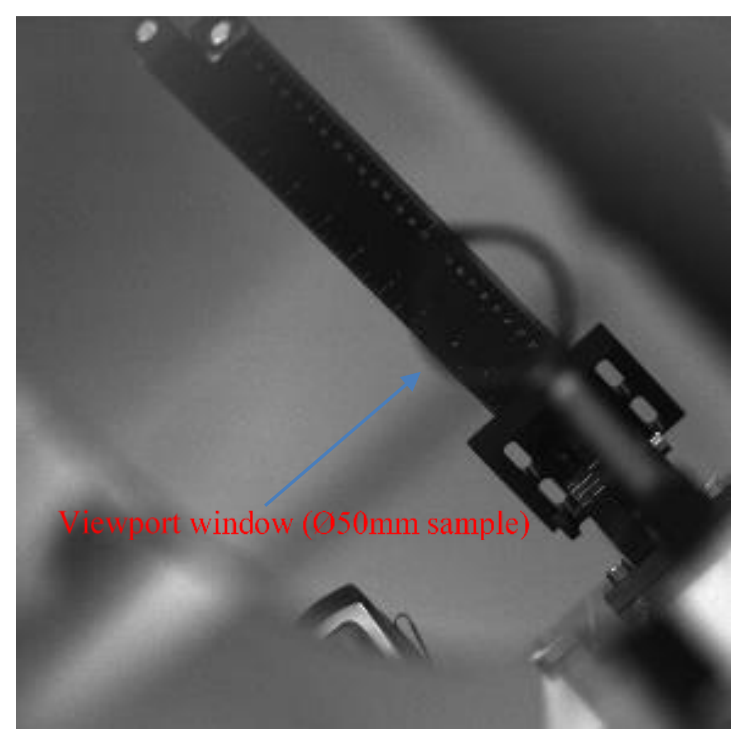

Figure 11 Image quality assessed by viewing a millimetre resolution test scale at the prototyping facility, through the same camera and lens as those proposed, plus a sample of viewport window.

Conventionally, the Modulation Transfer Function (MTF) is the standard measure of optical performance, imaging fine detail. However, a more realistic prediction of 
image quality than that simulated by Zemax has been obtained experimentally at the prototyping facility, equipped with a lens, mirror and camera identical to those planned for installation, as shown in Figure 11Error! Reference source not found.. In view just R. of centre is a small sam-

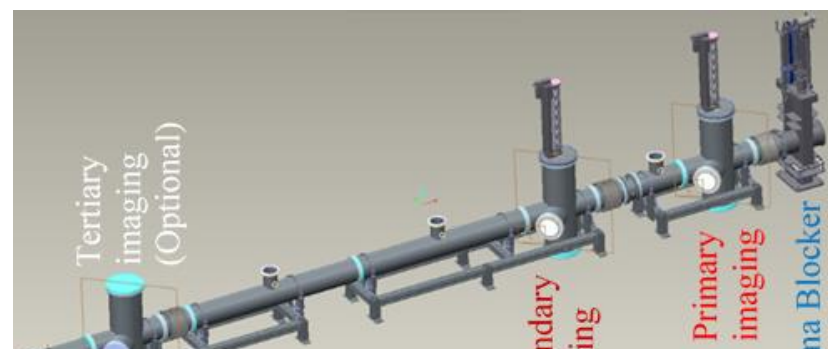

Figure 12 Locations of imaging stations in the dump line. Tertiary imaging vessel is to be initially installed empty. Beam direction is from bottom $\mathrm{L}$ to top $\mathrm{R}$.

ple representative of the proposed viewport window.

The limiting aperture in the system is the $200 \mathrm{~mm}$ diameter viewport, the mirrors being sized to accept the full field of view. The main optical properties of the chosen design are listed in Table 4Error! Reference source not found.; these are the values expressed in the Zemax model.

Table 4 Design parameters for the Tuning Dump system values entered into the Zemax model

\begin{tabular}{|c|c|}
\hline Parameter & TD system \\
\hline Focal Length $(\mathrm{mm})$ - set by lens selected & 135 \\
\hline f/\# $\{$ proposed $\}$ & $\mathrm{f} / 2.85\{\mathrm{f} / 2\} \dagger$ \\
\hline Mirrors: Clear Diameter (mm) & $\begin{array}{l}290 \\
110 \times 150\end{array}$ \\
\hline Screen - Lens Distance (mm) & $3828^{*}$ \\
\hline
\end{tabular}

*variable, depending on exact position of camera

$\uparrow$ depends on position \& hole diameter (see Figure 14)

\section{Tuning Dump System Components}

Initially, two identical systems will be installed, with provision for a third at a predefined upstream location, indicated in Error! Reference source not found.; access to three profiles would enable more advanced diagnostics, including emittance measurements.

\section{Mechanical System}
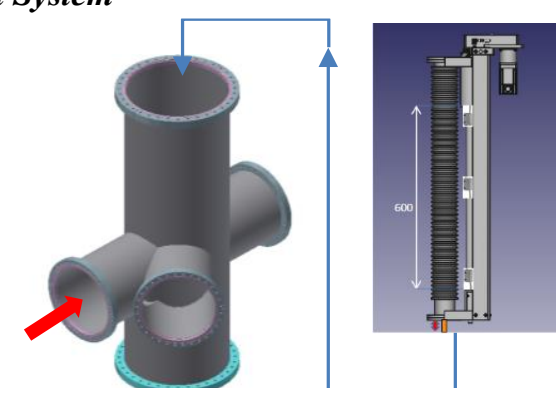

Figure 13 Imaging vessel final design (axiometric view). Beam enters from lower R., viewport flange to front. (Inset: Actuator showing limit switches.)
Each imaging vessel will be special custom-made 5-way cross, the horizontal arms conforming to the DN-250 standard of the beam-pipe, while the vertical will be to DN-350 to accommodate screens wide enough to allow coverage of the full aperture, illustrated in Error! Reference source not found.. With a beam-height of $500 \mathrm{~mm}$ above the tunnel floor, there is space below the beam-line for a vessel sized to accept the one unused screen in its lower vertical section, while the upper part is sufficiently tall to take both screens when fully raised clear of the beam. To facilitate maintenance reassembly, threaded 'half-moon' split-rings, rather than separate nuts (x36 for DN-350 size), will be used for clamping the top and the $45^{\circ}$ viewport flanges of the vessel; this also allows the flanges to be rotatable.

A long-travel vertical linear actuator on the top flange, seen in Figure 13, can move either of 2 screens into the beam. The actuator is a conventional edge-welded bellows design driven by a lead-screw. Special features include an in-line planetary gearbox to meet the torque requirement of $2 \mathrm{~N}-\mathrm{m}$ from a stepper motor with a moderate current load, though a directly-coupled option is also under consideration; the motor is selected for 'harsh-environment' (radiation) service. The configuration also avoids the need for an elastomeric drive-belt, which could be susceptible to radiation-induced failure; the annual dose to the motor as assessed by FLUKA modelling is 50 Gy. The system has no encoders, motion being controlled by five limit switches which sense intermediate screen positions as well as endsof-travel.

\section{Optical System}

Screens will be mounted at $90^{\circ}$ to the beam direction and will be viewed directly through a large diameter fusedquartz viewport on the vessel's $45^{\circ}$ arm. The viewport window may be changed when needed due to transmission loss; although quartz is radiation-resistant, FLUKA modelling predicts a dose of up to $5.7 \mathrm{kGy} /$ year.

Images will be relayed vertically through pairs of $45^{\circ}$ plane mirrors, to CCD cameras mounted in $1.5 \mathrm{~m}$ deep holes drilled in the tunnel walls, $1.5 \mathrm{~m}$ above beam height, as shown in Figure 14. Remotely-controlled lenses on the cameras will focus the final images and also provide for adjusting the $f$-number. Remote filter-changers located just in front of the lens, or alternatively at the entrance to the shield-wall hole leading to the camera, will provide selectable attenuation for image intensities which could, under certain beam-focusing conditions, exceed the dynamic range of the camera. It has also been demonstrated that depth-of-field across the full screen width may be improved by applying a tilt of less than $2^{\circ}$ to the camera sensor, using the Scheimpflug principle [16]. Characteristics of the selected optical elements are listed in Table 5.

An interface with the Machine Protection System, to inhibit the beam in case an unsafe condition is detected, will 
be provided through logic in the image processing software [17].

\section{Control Systems}

Controllers for both actuators and cameras will be located in racks outside the dump tunnel area. Motion control will use the ESS standard EtherCAT protocol which is supported by a range of commercially-available modules; these will also provide the logic processing for the limit switch array.

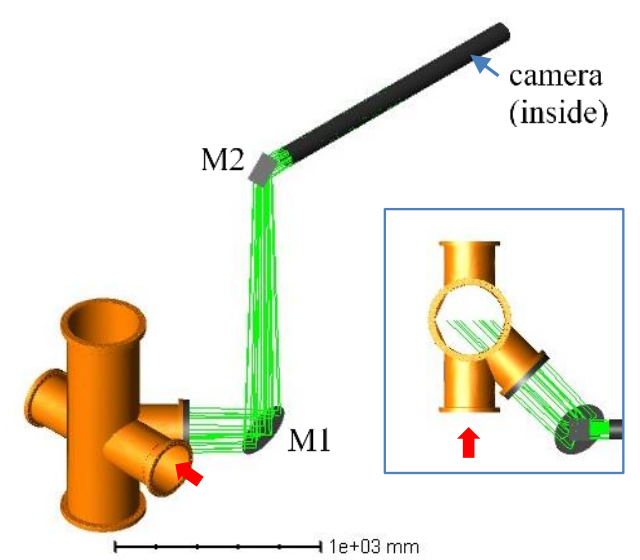

Figure 14 Optical path from a screen inside the vessel, via plane mirrors to a camera located inside a hole drilled into the shield wall. Proton beam direction is indicated by the red arrow. (Inset: Plan view, with beam from bottom.)

Table 5 Selection of Principal Optical Components. Mirror surface quality is quoted in wavelengths.

\begin{tabular}{|l|l|}
\hline \multicolumn{1}{|c|}{$\begin{array}{c}\text { Compo- } \\
\text { nent }\end{array}$} & \multicolumn{1}{|c|}{ Property } \\
\hline Mirror M1 & $\begin{array}{l}\text { UQG Optics 290mm diam x 6mm thick } \\
\text { Aluminium surface coated on borosili- } \\
\text { cate; } \mathrm{SiO}_{2} \text { protected } \\
\text { Quality: } 1 \lambda \text { per 25mm. }\end{array}$ \\
\hline Mirror M2 & $\begin{array}{l}\text { UQG Optics } 150 \times 110 \mathrm{~mm} \times 10 \mathrm{~mm} \\
\text { thick } \\
\text { Silver coated on UV fused silica; } \mathrm{SiO}_{2} \\
\text { protected } \\
\text { Quality: } \lambda / 4 \text { across surface } \\
\text { Hardness: 40-20 scratch-dig }\end{array}$ \\
\hline Lens & Canon 135mm f/2L USM \\
\hline Camera & $\begin{array}{l}\text { Allied Vision Manta G-419 } \\
\text { Sensor: CMOS 2048 x 2048 pixels }\end{array}$ \\
\hline
\end{tabular}

Figure 14 was generated in the 'non-sequential' mode of Zemax, which has also been used to explore the options for providing illumination of the screen in the absence of a beam. For this, it is currently proposed to locate a radiationhard light source between mirror M1 and the viewport.

\section{CONCLUSION}

An optical system has been designed to form images of the ESS proton beam profile at the end of the Tuning Dump line. The design has been developed and optimised to meet performance requirements under severe radiation environment constraints, and corroborated using the Zemax toolset, It has been shown that it is possible to adopt a very simple two-plane-mirror system while maintaining acceptable image quality; the cost and complexity of improving the image using further mirrors is not considered justified. Although detailed tolerancing studies for the effects of thermal expansion, misalignments, and production uncertainties are not presented here, prototyping of the system with mirrors of the specification to be installed has demonstrated they are able to meet imaging requirements.

Assessment of predicted radiation dose in the Tuning Dump line after irradiation has informed the location of imaging cameras for acceptable working lifetimes, the choice of materials for other key components, and the conditions to be expected during maintenance access. A detailed design for the imaging vacuum vessel and its mechanical elements has been developed to meet vacuum and other requirements.

\section{Applicability to Other Neutron Sources}

The design criteria have emphasised resilience and durability, giving assurance of longevity together with maintainability. As such, an imaging system of this type would be suitable for other high-power proton beamlines, unless a non-invasive diagnostic is required. The element of the system carrying the greatest risk is the screen itself, which is subject to beam damage, breakage or loss of emission, and this is mitigated by provision of a running spare at each imaging station.

\section{ACKNOWLEDGEMENTS}

The author would like to thank Scott Doyle and Ellis Chaffe for their invaluable assistance in compiling the analytical dose-rate data presented in Table 3.

\section{REFERENCES}

[1] Y. Lee, A. Olsson, M. Eshraqi et al., "Working Concept of $12.5 \mathrm{Kw}$ Tuning Dump at ESS", in Proc IPAC2017, Copenhagen, Denmark, paper THPVA065, pp 4591-4

[2] L Maxey, T Ally, A Brunson, F Garcia, T Shea et al., "A hybrid reflective/refractive/diffractive achromatic fiber-coupled radiation resistant imaging system for use in the Spallation Neutron Source (SNS)", Proc. Hard X-Ray, GammaRay, and Neutron Detector Physics XIII, Sept 2011, SPIE Vol. $8142,81420 \mathrm{~N}-1$

[3] ZEMAX LLC, Kirkland, WA 98033 USA, www. zemax.com/os/opticstudio

[4] D Korsch, "Reflective Optics", San Diego, USA: Academic Press, 1991, ISBN: 978-0-12-421170-4.

[5] M Gohran, R Linander, T Grandsaert, T Shea, C Thomas, "Proton Beam Imaging Options for the ESS Target", in Proc. IPAC 2014, Dresden, Germany, June 2014, paper THPME168, pp 3659-3661. 
[6] M Ibison, C. P. Welsch, E. Adli, H. Gjersdal, N. de la Cour, T. Shea, C. Thomas, "Optical System Design for THE ESS Proton Beam and Target Diagnostics", in Proc. IPAC 2016, Busan, Korea, paper MOPMR043, pp 347-349

[7] E. Adli, H. Gjersdal, O. M. Røhne, C. Thomas, T. Shea. R. Andersson, M.G. Ibison, C.P. Welsch, S. Joshi, "The Ess Target Proton Beam Imaging System as In-Kind Contribution" in Proc IPAC2017, Copenhagen, Denmark, paper WEPVA066, pp 3422-4

[8] M Donna, T Grandsaert, M Göhran, R Linander, T Shea et al., "Optical System for ESS Target Protection", in Proc. IBIC 2014, Monterey, CA, USA, Sept 2014, paper TUPF27

[9] A. Ferrari et al., "FLUKA: a multi-particle transport code", CERN-2005-10, INFN/TC_05/11, SLAC-R773, 2005.

[10] S. Hutchins, M. Facchini, E. Tsoulou, "Radiation Tests on Solid State Cameras for Instrumentation", Proc. DIPAC 2005, Lyon, France

[11] R. Michel, M. Gloris, H.-J. Lange et al, "Nuclide production by proton-induced reactions on elements $(6 \leq Z \leq 29)$ in the energy range from 800 to $2600 \mathrm{MeV}$ ", Nuclear Instruments and Methods in Physics Research B 103 (1995) 183222

[12] Bruce C. Brown, Nikolai V. Mokhov and Vitaly S. Pronskikh, "Activation Products from Copper and Steel Samples Exposed to Showers Produced by $8 \mathrm{GeV}$ Protons Lost in the Fermilab Main Injector Collimation System", Fermi National Accelerator Laboratory, Batavia, IL, USA

[13] "Dose Calculations: Absorbed Dose from a charged particle beam", MIT Course in Nuclear Engineering, Principles of Radiation Interactions 22.55 (2004)

[14] Ana Neacsu, Mihail Contineanu, Traian Zaharescu, Iulia Contineanu, "Calculation of the Gamma Radiation Dose Produced by a Cylindrical Radioactive Source", REV. CHIM.(Bucharest) 67 No. 92016

[15] M. Turner, B. Biskup, S. Burger, E. Gschwendtner, S. Mazzoni, A. Petrenko, "The two-screen measurement setup to indirectly measure proton beam self-modulation in AWAKE", Nuclear Instruments and Methods in Physics Research A 854 (2017) 100-106

[16] T. Scheimpflug, "Improved Method and Apparatus for the Systematic Alteration or Distortion of Plane Pictures and Images by Means of Lenses and Mirrors for Photography and for other purposes", UK Patent No. 1196, Jan 1904

[17] T Shea, L Coney, R Linander, A Jansson, E Pitcher et al., "Instrumentation \& machine protection strategy for the ESS target station", in Proc. ICANS XXI, Mito, Japan, Sept 2014 